\title{
Factors determining dropping out from treatment among methadone clients: a qualitative study in Can Tho City, Vietnam
}

\author{
Nguyen Ai Hong, Zwanikken Prisca, Ho Hien, Dinh Cong Thuc, Nguyen Trong Nhan \\ Can Tho Provincial HIV/AIDS Control, Can Tho City, Vietnam
}

\begin{abstract}
Introduction: Methadone maintenance therapy (MMT) is a multiple therapy used for opiate dependence. It is combined with psychological therapies that aim at supporting individuals, families, and communities in improving their health, reducing criminal activities, and minimising risky behaviours for human immunodeficiency virus (HIV) transmission. Retention in treatment is associated with a successful outcome of MMT.

Material and methods: Provided since 2008 in Vietnam, MMT has been implemented in Can Tho province since 2010. However, its dropout rate is high: nearly $40 \%$ from 2010 to 2015 . Evidence is insufficient to understand the factors that influence the drop out rates among MMT patients in Vietnam, and more especially in Can Tho province. There is an urgent need to better understand the reasons and the context in which MMT dropouts occur, in order to inform the development of policies and programmes that can improve adherence to MMT in Can Tho.

Results: Qualitative interviews have been conducted with 17 methadone clients (dropouts and current), 4 health workers and 3 family members of clients. Barriers to retention in treatment included individual factors, community factors and institutional factors.

Conclusions: The factors influencing the decision to drop out among MMT clients could be at individual, community, or institutional levels. This study suggests that increasing meaningful involvement of the clients' families and strengthening counselling sessions on adherence for clients can improve their retention in treatment and thus the MMT outcomes.
\end{abstract}

HIV AIDS Rev 2017; 16: 32-39 DOI: https://doi.org/10.5114/hivar.2017.65235

Key words: methadone maintenance, drop out, Can Tho, Vietnam, people who inject drugs, drug users.

\section{Introduction}

Vietnam's human immunodeficiency virus (HIV) epidemic is concentrated among the high-risk behaviour population such as female sex workers (FSW), people who inject drugs (PWID) and men who have sex with men (MSM). HIV prevalence among PWID was reported to be as high as $10.3 \%$ compared to under $0.3 \%$ among the gen-

eral population [1]. In Vietnam, the estimated number of PWID was 270,000 people in 2013. Of the 224,000 people living with HIV (PLWHIV) in 2013, 39\% were PWID. Methadone maintenance therapy (MMT) has been initiated in 2008 in Hai Phong and Ho Chi Minh City [1]. By 2013, MMT programmes were implemented in 88 clinics, covering 33 (out of 63) provinces, and serving 15,542 PWID [1].

Article history:

Received: 01.09.2016

Received in revised form: 14.11.2016

Accepted: 15.11.2016

Available online: 19.01.2017
International Journal of HIV-Related Problems

HIV \& AIDS

R e vi e w 
Can Tho is one of the provinces that belong to the Mekong river delta area of Vietnam. The province has 1.2 million inhabitants, of whom $49.6 \%$ were male in 2013 [2]. This province is divided into 4 districts and 5 quarters.

Regarding the HIV epidemic, Can Tho is one of the cities in Vietnam where the HIV prevalence has been ranked as high. The cumulative number of people living with HIV was 5458 in 2014, with a HIV prevalence among PWID of $24 \%$. It is estimated that there were around 2000 PWID in Can Tho. MMT programmes started to be implemented in Can Tho City 5 years ago, in 2010. According to the PAC of Can Tho in 2014, the cumulative dropout rate from treatment between 2010 and 2014 was $44 \%$ [3].

Methadone is a medication used to treat opiate dependence. It is often combined with psychological therapies that aim at supporting individuals, families, and communities in improving their health, reducing criminal activities, and minimising risky behaviours for HIV transmission [4].

Dropping out from treatment can lead to a relapse into drug injection and changes in behaviour. Furthermore, needle sharing practices among dropouts have also increased [5]. Reasons for dropping out from MMT treatment which are known in the literature are related to methadone dose, duration of treatment, gender difference, and history of opioid dependence [6].

A number of studies have been conducted among methadone clients in Can Tho City [7, 8]. However, none of those studies have explored the factors of dropping out from treatment among methadone clients. Therefore, there is an urgent need to better understand the reasons why MMT dropouts occur, in order to inform the development of policies and programmes that can help improve the adherence to MMT in Can Tho.

\section{Material and methods}

\section{Sample}

Convenience sampling was used to recruit 17 participants for this study. Inclusion criteria were the following: being 18 years of age or older, having civil abilities, and agreeing to participate in the study.

- Current MMT participants: were contacted by the administrators of the MMT clinics, as administrators have to contact their current clients regularly to remind them to come and take their drugs. After consenting to participate in the study, the participants were contacted by the researcher in order to schedule an interview.

- Dropouts: recruited by peer educator group. This recruitment method has been chosen because evidence has shown that the relapse rates among dropouts is high, and therefore peer educators of a harm reduction programme are key persons to reach dropouts. Indeed, it has been estimated that $78 \%$ of PWID are reached by the peer educator group through harm reduction programmes [3]. In a meeting with the peer educators' group, the objectives, methods and selection criteria for the participants were introduced. Then, the research team asked the peer educators to invite the dropouts they already know to join the study. After reaching an agreement with the dropouts, the peer educators informed the research team about the time and location to conduct in-depth interviews.

- Health workers in MMT clinics have been informed by the Can Tho Provincial HIV/AIDS Control and clinics. Doctors and administrators were interviewed.

- Relatives of MMT clients were chosen based on the client's preferences. The research team then contacted the chosen relatives to schedule the in-depth interviews.

\section{Data collection and data analysis}

Data were collected with in-depth interviews with key informants. The structure of the interview guide was divided into three levels of reasons for dropping out from treatment: individual, community, and institutional (Table 1).

The interviews were tape-recorded and transcribed verbatim. MS Excel was used to organise the transcript. The data were then coded based on specific objectives and themes which were identified in this study, mainly the explanation of the difference between the current MMT clients and the dropouts. Data analysis was conducted through 3 stages: completion and management of data, analysis, and report writing. Both data collection and analysis were conducted in Vietnamese, while the report was written in English.

\section{Results}

\section{Factors determining drop out from treatment}

\section{Individual factors}

Occupation: The characteristics of the job and financial catastrophes could be influenced to stay longer on treatment, especially for those who had mobile jobs. Another reason was that, due to the purchase of heroin and living expenses, some of them had to become a debtor, so they had to run away from the creditors.

"Because I had to a move to a rural area for my work,

I could not access the methadone treatment. We are not allowed to bring methadone doses home and the methadone drug is liquid so it is difficult to bring it home, so I could not follow up the treatment." (Drop out client)

"Due to their debt, they had to run away from creditors because they could not have reimbursement. Hence, they had to give up treatment." (Health worker - Female)

The knowledge related to HIV and MMT: An inadequate knowledge about MMT and the risk of HIV transmission could lead to underestimating the role of MMT services and to engaging in risky behaviour for HIV transmission. Both dropouts and health workers shared the idea that misunderstandings and fear of side effects of methadone could contribute to drop out from treatment:

"Some methadone clients thought that heroin was more satisfying than methadone. Other clients said that after 3 years 
Table 1. Theoretical topic guide

\begin{tabular}{|c|c|}
\hline \multicolumn{2}{|l|}{ Individual factors } \\
\hline Knowledge related to HIV transmission and MMT & $\begin{array}{l}\text { Can you tell me the way of HIV transmission? } \\
\text { Do you think that you have a risk of acquiring HIV/AIDS? } \\
\text { How and why? } \\
\text { What are the roles of MMT? } \\
\text { The results of dropping out from MT treatment? }\end{array}$ \\
\hline Individual factors could lead to drop out from treatment & $\begin{array}{l}\text { Which individual reason for dropping out from treatment? } \\
\text { Example } \\
\text { - Financial issues } \\
\text { - Occupation requirements } \\
\text { - Self determinations } \\
\text { - Fear of side effects of methadone drug } \\
\text { - Transportations } \\
\text { - Self stigmatization }\end{array}$ \\
\hline Community factors & $\begin{array}{l}\text { Which reasons from the community lead to drop out from } \\
\text { treatment? } \\
\text { Example } \\
\text { - Family support } \\
\text { - Peer influence } \\
\text { - Stigma and discrimination from community }\end{array}$ \\
\hline \multirow[t]{2}{*}{ Institutional factors } & $\begin{array}{l}\text { Which reason from institutional factors could lead to drop } \\
\text { out from treatment? } \\
\text { Example } \\
\text { - The rules of health setting } \\
\text { - The daily oral dose of methadone drug } \\
\text { - The working time of the clinic } \\
\text { - The distance from home to the clinic } \\
\text { - The attitude of health workers } \\
\text { - The waiting time to get the dose in the clinic }\end{array}$ \\
\hline & $\begin{array}{l}\text { Which factors do you think should be improved to help } \\
\text { clients stay longer on treatment? }\end{array}$ \\
\hline
\end{tabular}

of treatment, they could give up heroin and were successful in their methadone treatment, so that they dropped out without the permission of the doctors." (Health worker Female)

Regarding their knowledge about HIV/AIDS, both dropouts and current clients knew about HIV transmission. Nevertheless, almost all of them believed that they were not at high risk of being infected by HIV.

"I do not think I am at risk of getting HIV because I did not share the needle and injection materials with any other drug users." (Current treatment client - Man)

Inadequate knowledge can be due to lack of information sources and unavailability of information channels related to HIV/AIDS and MMT. Common channels used by clients and their relatives were television, internet, health workers, and committee authorities. All participants shared a similar idea of the consequences of leaving the treatment against the health workers' decision: relapse in heroin usage, and appearance of health issues such as weight loss or insomnia.

Clients' self-determination: Self-determination seemed to be a motivation to stay longer on treatment. Indeed, visiting MMT clinics every day can affect the clients' patience.
Also, giving up heroin is not easy and progress takes time. Moreover, according to the MMT guidelines, all clients have to start the treatment with a methadone dose of $20 \mathrm{mg}$, so it is likely that the clients will not be satisfied at the dosefinding stage. The decision to give up heroin seemed to play a vital role regarding how long clients would keep on attending MMT:

"Due to a lack of determination for treatment and the fact that they thought they could not get high or satisfied with the methadone dosage, some of them gave up at the dose-finding phase, even if they attended the pre-treatment counselling and even if all the information related to MMT was provided to them." (Health worker - Female)

Besides that, depression in some clients who were HIV positive could have led to dropping out from treatment. Because HIV cannot be cured, some thought that they would die soon anyway and might as well enjoy their last days with heroin. They also thought that if they take up both ARV and methadone treatment, they will then get "drug poisoning".

"The clients who are HIV positive, if they take the methadone drug they will die soon." (Dropout client - Man) 
Unreadiness for treatment: Unreadiness for treatment could also be a factor influencing drop out from treatment. In Vietnam, the PWID, because of their use of drugs that are illegal in the country, will be under observation of the police or the community authority. If a PWID joined MMT, the observation by the police would be reduced. In other words, MMT can become a shield for clients who want to avoid observation. Under this protection, the MMT clients might actually not give up on their heroin injections. Some of them even got arrested by the police because of criminal activities.

"Some clients were suspected by the police or were under the management of the commune authorities because of illicit drug use. Hence, they attended MMT without wanting to get treated." (Health worker - Female)

Reason for health-seeking behaviours: Both dropouts and current clients said that the main reason for using MMT was to change their lifestyle from heroin injection, which they called "abnormal life", to "normal life", which is good health for a healthy life. They and their family felt tired of the heroin-injection life style and the related financial catastrophes. Despite that, if the reason for treatment was legal pressure, then clients tended to leave treatment earlier.

"I want to give up on the behaviours related to heroin injection. Before attending MMT, I visited some heroin detoxification centres several times but without success. Being a heroin user, I had to steal goods from other people to get money for heroin. I do not want to have a life like this anymore." (Current treatment client - Man)

Gender norm: Cultural norms are a pressure for MMT clients. This could be linked to their personal roles in their families, and gender-based stereotypes. During the in-depth interviews, male clients tended to say that they had to earn money for their family, while one of the female dropouts said that she could not stay longer on treatment because she had to take care of her family. The gender-based stereotypes underlying these statements are that males should go out and earn money for their families and that females should take care of their families. The decision to drop out seemed to be related to the individual responsibility they took:

"Sometimes I think that now that I have children and a husband to take care of, I do not want to follow up the treatment for a long time." (Drop out client - Female, 32 years old)

For a male dropout, being a father made him not want to harm his family:

"Because of my family and my child, I had to give up the treatment because I did not want rumour to be brought to my child. What would people think if they knew his father was an addict who has to take methadone every day?" (Drop out client - Man, 38 years old)

\section{Community factors influencing drop out from treatment among MMT clients}

Peer influence: Peers can influence the MMT clients in two ways: by encouraging them, or by constraining them. Current MMT clients explained that they got to know MMT from their friends and that the latter also advised them to stay on treatment longer. Health workers also confirmed these facts. All participants said that familial support plays a vital role for clients to stay longer on treatment. The kinds of familial support that were mentioned were financial support, providing employment, support by helping with transportation, and mental support. Being disregarded by their family members could lead the MMT clients to depression, which can in turn lead to drop out from MMT:

"I heard about MMT from some friends, who were PWID.

They had attended the treatment before, so they con-

vinced me to join them." (Current treatment client - Man)

On the other hand, some clients' relatives affirmed that these peers encouraged their son or their husband into relapsing with heroin injection, and that this was the reason why they wanted to keep them away from these peers:

"Almost all of my husband's friends are PWID, so I tried to keep him away from them as much as I could. I know that some of them asked my husband to relapse into heroin injection, but he refused." (Relative's client - Female)

Stigma and discrimination: Stigmatisation and the fear of being stigmatised by the community constitute one of the obstacles to stay longer on treatment. PWID are usually familiar with stigma and discrimination because they suffered from it with the people who are living around them:

"I am a man; I have to work to earn money for my life and for my family. If they know that I am an MMT client, which means that I am a PWID, they will look down on me and my kids will be isolated from their friends because their father is a PWID." (Drop out client - Man) Still on the theme of stigma and discrimination, all participants said that almost all PWID had suffered from stigma and discrimination. Moreover, they also explained that their family could also become a victim of stigma and discrimination:

"People look down on me and my family. I could feel the stigma, even if they did not talk. It is from their attitude toward me." (Relative's client - Female)

Family support: Pressure from the family influenced the clients' decision to go on treatment. A client's relative said that the pressure from the family helps the clients understand what is good for them. Therefore, under the pressure from the family, especially under the one of being a "good father" or a "good son", clients stayed longer on treatment.

From the clients' side, the pressure from family can lead to unwillingness to get treated. They would just come and take the drug under the pressure from their family, without really wanting to stay longer on treatment:

"At the beginning, my parents pushed me to attend the treatment and they also forced me to take methadone. But I was not willing to get treated, I thought that methadone and heroin were the same." (Drop out client - Man) Inter-sectoral efforts at the community level are seen as a way to reduce the dropout rates from treatment and thereby increase the retention rate on treatment among MMT clients:

"We do need inter-sectoral efforts to mobilise the PWID to attend treatment and stay longer on it. The police should force the PWID to attend the treatment. Besides 
that, we do need to have some career policy that helps clients have a stable life and a permanent job." (Health worker - Female)

\section{Institution factors influencing drop out from treatment}

Daily methadone dose: The daily dosage can influence the drop out from treatment among MMT clients. According to the guidelines, the clients have to take a dose of methadone every day under the health workers' observation. Almost all participants said that the dosage of daily oral methadone influenced their decision to drop out. Indeed, attending the MMT implied that, during their treatment time, the clients could not travel outside of the city and felt like their independence and freedom were restricted. Also, at the finding-dose phase of the treatment, the methadone doses were also not strong enough to stop their craving for heroin.

Nevertheless, all the participants shared the view that the amount of methadone they were given at each dose did not affect their decision to drop out or to adhere to the treatment:

"Methadone dose did not affect retention on treatment or drop out from treatment, it was based on the selfdetermination whether they want to use heroin or not. For me, my methadone dose was $140 \mathrm{mg}$ per day but I was still using heroin." (Drop out client - Man)

Open time of clinic: Additionally, an inappropriate working time could also lead to drop out from treatment. The opening hours of health facilities coincided with the working time of the clients who were employed. They could therefore not leave their working place every day during their shift to attend their treatment at the MMT clinic:

"Due to the overlap between my working shifts in my company and the time of methadone dose uptake, I could not stay longer on treatment even though I wanted to attend it. I am telling you that if the clinic had been open during non-working time, I would not have given up my treatment." (Drop out client - Male)

In order to reduce the drop out related to inappropriate working time, the MMT clients suggested that the clinic should be open outside of regular working time (7 a.m. to 5 p.m.) to help its client to access their treatment. A health worker added that the procedure of delivering methadone should be simplified and be made more flexible:

"The opening time of methadone clinics was inappropriate. My working time starts at 7 a.m. and the time of my appointment for methadone was also at 7 a.m. So I could not continue working if I was on treatment." (Drop out client - Male)

Long waiting time for starting treatment: The people who register for MMT must wait before getting the treatment. A long waiting time before treatment could also influence the decision to drop out among MMT clients. From the data collected from the clients' records, the mean waiting time for treatment was 53 days. After registration, some individuals dropped out because the waiting time was too long.
However, the waiting time has been reduced since, with the newest treatment procedure:

"The current treatment procedure is a new one, and the procedure has been simplified. In the past, clients had to wait for confirmation from the commune before being allowed to attend the treatment. Now, with the application of the new policy, clients wait for less than 10 days before initiation of treatment." (Health worker - Female)

Attitude of health workers: Negative attitude was considered as a kind of stigma. Because they were addicted to heroin, MMT clients suffered from negative attitudes, not only in their community, but also from health settings.

The attitude that the clients and the health workers have toward each other was indeed mentioned as a factor that could lead to drop out from treatment. The clients felt they were looked down on by the health workers:

"Because of the discrimination due to being a PWID, I decided to attend the treatment. However, when I was on treatment, the health workers looked at me as if I was a robber or an uneducated person. I have self-esteem and I am educated, I did not break the law, so why do they have this attitude toward me?" (Drop out client Man)

Long distance from home to clinic: Health workers and current MMT clients shared similar views about the distance between home and the MMT clinic being a barrier to adherence to treatment. In O Mon clinic, clients come from other district to get their dose. The long distance and the lack of means of transportation to go to the clinic every day could lead to non-adherence and drop out from the treatment. One of the clients said that:

"The lack of vehicle can lead to dropping out because the clients don't have enough money to access clinics and almost all the clients are so poor they don't have their own motorbike to go to the clinics." (Currently on treatment - Female, 27 years old)

Unclear treatment progress: Long treatment duration seemed to be confusing for clients. Having to spend more than a year to give up on drug dependence was considered to be too long for some people. For example, when being sick, people go to the doctor and know how much time their recovery will take. It is different with MMT.

From the perspective of a client's relative, the unclear treatment procedure that could vary between different clients can make the clients depressed. Consequently, they could not stand the idea of staying in the treatment any longer:

"My daughter has been attending the treatment for 4 years, but I don't know when she will finish her treatment. The doctors must inform the clients about the treatment procedure. For instance: a creative treatment procedure for each client with which she will be done after 4 or 5 years, with a clear process for dose reduction. I saw my daughter. She had to take methadone doses of $20 \mathrm{mg}$ per day for a long time. Why have the doctors not given her the reducing dose? These things made us tired." (Relatives' client - Female) 


\section{Discussion}

\section{Individual factors}

Adherence during treatment: According to the findings, decreased adherence among dropouts may be due to their self-assessment. After a long time of treatment, clients may think that they can give up heroin injection and that their treatment was successful. In consequence, adherence can be reduced. This idea was mentioned by health workers and current clients.

The study conducted in London to explore the pattern of non-adherence shares similar results: a small majority of the current clients were "adherent" to the treatment (58\%), $24 \%$ of them were "partially adherent" and $18 \%$ of them were of "poor adherence" [9]. In the study of Haskew, the level of adherence was categorised into three scenarios: "poor adherence" means that clients had consumed 1-2 methadone doses per month, "partial adherence" was the group of clients who consumed 3-28 doses of methadone per month, and "adherence" means that client who were consumed 29-30 doses of methadone per month.

Community factors: Almost all participants said that support from their family and their relatives could be a factor influencing drop out from treatment. Family could be a motivation for retention on treatment.

Family support implies both mental and financial support. This is in line with the results of a cross-sectional study among 590 methadone clients that explored the relation between family support and the outcome of a methadone programme. The clients who received the support from family were less likely to keep using illicit drugs than those who were not supported by their family $(p<0.01)$ [10]. Currently, the involvement of family during the treatment is voluntary. Consequently, it might not be well coordinated with the clinics in their efforts to help clients stay longer on treatment and have a successful treatment.

The personal perspectives shared during the interviews of this study revealed that the influence from peers could be either negative or positive for the retention on treatment. According to the results of the study that took place in China among PWID who attended methadone treatment, the rates of retention to treatment were lower among the clients who had PWID as friends, compared to the clients who did not have this kind of friend $(p<0.005)$ [11]. The rates of heroin usage were also lower among those who had family support, compared to those who did not have support from their family $(\mathrm{OR}=0.97, p<0.01)[10]$.

\section{Institutional factors}

Daily oral dose: A daily liquid dose of methadone taken under the observation of a health worker is one of the requirements for running a methadone clinic. However, from the literature review, we learnt that Stark identified the dosage of methadone as an institutional factor influencing the reten- tion on treatment. Here, receiving methadone in daily oral doses seemed to be a barrier to retention on treatment.

Consuming methadone at the clinics every day could lead to reduced freedom of clients, so it could be a constraint for a longer treatment duration. Participants suggested ideas such as receiving methadone at home, or being allowed to take their dose home.

A retrospective cohort study was conducted in Italy. In this study, researchers compared two groups: one that had daily doses in the clinic, and another that was taking their dose at home and went to the clinic only once a week for a health check-up. Here, the results were different: the clients taking their dose at home had significantly higher rates of leaving the treatment for detoxification (23.2\%), compared to the group who took daily doses of methadone in the clinic $(3.6 \%)[12]$.

Taking doses at home can therefore be an obstacle to treatment. Nevertheless, this can still be a good way to help clients organise their daily life and avoid daily attendance in the methadone clinics. A daily visit to a methadone clinic could be a factor influencing retention of clients. Findings also showed that clients tended to stay longer on treatment when receiving high methadone doses ( $\geq 60 \mathrm{mg}$ ) compared to those who got low doses $(\leq 40 \mathrm{mg})$ [12].

When looking at the association between the dose of methadone and heroin consumption, a higher dose of methadone could actually be a predictor of heroin usage during the treatment. Indeed, Luu Hoang Viet found that the prevalence of heroin usage among the group of clients who consumed a methadone dose $\geq 80 \mathrm{mg}$ was 3.66 times higher than the group of those who received a methadone dose $\leq 80 \mathrm{mg}$ [7].

Research conducted among Malaysian methadone clients in 2010 also found that the daily dose of methadone could contribute to the retention of the clients. Here as well, the retention prediction of clients who received a dose of more than $80 \mathrm{mg}$ was higher than those who received a daily dose less than $80 \mathrm{mg}$. The required dose for retention on treatment is $40 \mathrm{mg} /$ day. Nevertheless, and in line with the above-mentioned studies, Mohamad recommended that a dose of $80 \mathrm{mg}$ was the most likely to lead to successful treatment [13].

Distance from home to the clinic: The distance from home to the clinic seemed to be a barrier to accessing the services, and it can also influence the decision to drop out among clients. This was the case in O Mon clinic, where more clients came from other districts to attend the treatment.

A study conducted by Greenfield et al. with 1753 clients attending methadone treatment in an urban area found that the clients who travelled less than 1 mile to reach the clinic were more likely to stay longer on treatment than those who had to travel more than 1 mile. The retention rates among mobile methadone facilities were higher than those in fixed methadone facilities. Indeed, with a mobile facility, both time and cost of transportation are reduced for the clients, and it therefore leads to greater service accessibility [14].

However, the distance from home to the clinic should be put into the context of whether public transportation is 
available. If the distance is far but transportation is available and the travel fee is reasonable, a long distance might not be a barrier, neither for accessing the services nor for retention on treatment. In the context of this study, public transportation was not available, which could have been a constraint to stay longer on treatment.

Treatment progress: Unclear treatment progress for individual clients could lead to early leaving of treatment. This notion appeared during in-depth interviews. Clients were tired with the time their treatment took because it was time-consuming and they did not have any information about their progress. They needed to know when they would finish their treatment and wanted to see the treatment plans the doctors prepared for them.

Perterson et al. conducted a study among clients who dropped out in Baltimore, Maryland, with the purpose of exploring the reason why PWID were dropping out of methadone treatment. The barriers included a long waiting list, lack of health insurance, fear of side effects of methadone, and various requirements in the registration procedure [15].

Compared to the findings from our research, there was a concordant finding: unclear treatment progress could lead to drop out. Even though the quantitative part only focused on clients who had already dropped out, similar barriers related to the treatment progress were found to be leading to drop out, both during treatment and at a pre-treatment time.

Ball et al. conducted their study among 24 clients who had dropped out. The researchers developed a questionnaire in which different reasons to drop out from treatment were arranged into categories. Their results shared similarities with our study: transportation fees, the relationship between the clients and the health workers, their family relationships, and their individual views on the programme were factors influencing their decision to drop out from their treatment. However, in their study Ball et al. also found that the fee for services in MMT clinics and stigma and discrimination were also predictors for dropping out of treatment, which was the case in our study. The difference is that in the context of Vietnam, MMT is offered for free to all clients. Therefore, a fee for services was not a predictor of drop out. As to discrimination and stigma, they seem to be reasons for leaving the treatment, even though these phenomena have been experienced by some of the participants of our study [16]. Issues in the clients' health-seeking behaviour and finances could also be factors affecting their decision to drop out. In a cohort study conducted in Hai Phong and Ho Chi Minh City, the reason to drop out among $43 \%$ of the methadone clients was arrest (FHI), which was a higher percentage than the figures that resulted from our research (36\%) [17].

\section{Limitations and strengths of the study}

The fact that convenience samples were used introduces a selection bias. Also, dropouts who have been arrested could not be reached by this study.
This study fails to explore other factors related to dropouts that are not mentioned in the topic guide, such as cultural factors and gender issues.

Regarding the convenience sampling, other criteria for purposive sampling are potentially relevant to gaining a wide insight into the reasons behind dropping out or staying on treatment, for example: one could distinguish between the rich/poor family PWID, young/old PWID, male/ female PWID, HIV status, etc. Each of these criteria could form the basis for purposeful sampling - but given the constraints of time and resources, I have limited the scope of my enquiry, which may have therefore influenced the results.

This study met its objectives: the reasons to drop out from MMT were explored. The sample size of the study was adequate for a small exploratory case study research proposal. Criteria in terms of gender and number of participants were met.

\section{Conclusions}

The factors that could influence drop out from MMT were: inadequate knowledge about methadone treatment and methadone dose, occupation-related and financial barriers, lack of family support, fear of stigma and discrimination, long distance from home to the clinic, unclear treatment progress, daily oral dose of methadone, adherence during treatment, legislation of methadone clinics, and the attitude of health workers toward clients.

\section{Acknowledgements}

This research was approved by the Research Ethics Committee on Proposal S63 in the Royal Tropical Institute, The Netherlands and Ha Noi School of Public Health, Vietnam.

We acknowledge Dr. Sumit Kane and Monique Tan for their support and providing many meaningful comments on this study.

The results of this study were defended for a master thesis both in Ha Noi School of Public Health and the Royal Tropical Institute.

\section{Conflict of interest}

The author's declared no potential conflicts of interest with respect to the research, authorship, and/or publication of this article.

\section{References}

1. Xuan Phuc N. Viet Nam AIDS Response progress report 2014 - following up the 2011 political declaration on HIV/AIDS - Reporting period 2012 - December 2013, Ha Noi, 2014.

2. General statistic offcer, 2013. Statistical handbook in 2013, Ha Noi.

3. Can Tho Provincial AIDS control. The report on results of HIV/ AIDS program in 2014. Can Tho, 2014.

4. Moh BY, et al. The methadone treatment guideline. Ha Noi, 2010.

5. Ball JC, Lange WR, Myers CP, et al. Reducing the risk of AIDS through methadone maintenance treatment. J Health Soc Behav 1988; 29: 214-226. 
6. Stark MJ. Dropping out of substance abuse treatment: A clinically oriented review. Clin Psychol Rev 1992; 12: 93-116.

7. Hoang Viet L. The results of Methadone treatment program in Ninh Kieu district, Can Tho city from 2010 to 2013. Can Tho Medical University, 2014.

8. Thanh Nam D. The result on implementing Methadone treatment programe in Cai Rang district, Can Tho City from 2010 to 2013. Hanoi School of Public Health 2014 [In Vietnamese].

9. Haskew M, Wolff K, Dunn J, et al. Patterns of adherence to oral methadone: Implications for prescribers. J Subst Abuse Treat 2008; 35: 109-115.

10. Lin C, Wu Z, Detels R. Family support, quality of life and concurrent substance use among methadone maintenance therapy clients in China. Public Health 2011; 125: 269-274.

11. Booth RE, Corsi KF, Mikulich-Gilbertson, et al. Factors associated with methadone maintenance treatment retention among street-recruited injection drug users. Drug Alcohol Depend 2004; 74: 177-185.

12. Pani PP, Pirastu R, Ricci A, et al. Prohibition of take-home dosages: Negative consequences on methadone maintenance treatment. Drug Alcohol Depend 1996; 41: 81-84.

13. Mohamad N, Bakar NH, Musa N, et al. Better retention of Malaysian opiate dependents treated with high dose methadone in methadone maintenance therapy. Harm Reduct J 2010; 7: 30 .

14. Greenfield L, Brady JV, Besteman KJ, et al. Patient retention in mobile and fixed-site methadone maintenance treatment. Drug Alcohol Depend 1996; 42: 125-131.

15. Peterson JA, Schwartz RP, Mitchell SG, et al. Why don't out-of-treatment individuals enter methadone treatment programmes? Int J Drug Policy 2010; 21: 36-42.

16. Ball S, Carroll KM, Canning-Ball M, et al. Reasons for dropout from drug abuse treatment: Symptoms, personality, and motivation. Addict Behav 2006; 31: 320-330.

17. USAID and FHI360. Effectiveness evaluation of the pilot program of opioid dependence with methadone in Hai Phong and Ho Chi Minh cities (after 12 month treatment). 